\title{
Correlación entre los grosores de las capas de células ganglionares y de fibras nerviosas temporal, en sujetos sin y con diabetes
}

\section{Correlation between temporal ganglion cell layer and nerve fiber layer thickness, in diabetic and non-diabetic patients}

\author{
Iván Salgado-Flores ${ }^{1}$, Dulce M. Razo Blanco-Hernández², Selma A. Somilleda-Ventura ${ }^{3}$ y \\ Virgilio Lima-Gómez ${ }^{1 *}$
}

${ }^{1}$ Plan Único de Especializaciones Médicas en Oftalmología, Hospital Juárez de México; ²División de Investigación, Hospital Juárez de México; ${ }^{3}$ Centro de Investigación Biomédica, Fundación Hospital de Nuestra Señora de la Luz. Ciudad de México, México

\begin{abstract}
Resumen
Objetivo: Determinar la correlación entre los grosores de las capas de células ganglionares y de fibras nerviosas retinianas en el sector temporal, en sujetos con y sin diabetes. Diseño: Observacional, retrospectivo, comparativo y transversal. Material y métodos: Se estudiaron sujetos sanos y diabéticos sin retinopatía, de entre 20 y 80 años de edad. Se midieron mediante tomografía de coherencia óptica los grosores de las capas de células ganglionares y fibras nerviosas en el sector temporal. Se compararon sus promedios entre grupos (t de Student) y se determinó la correlación entre ambas variables en cada grupo ( $r$ de Pearson). Resultados: 127 ojos fueron evaluados, 53 ojos de sujetos sanos y 74 de pacientes con diabetes sin retinopatía. Los grosores de las capas de células ganglionares y de fibras nerviosas no difirieron entre grupos. En sujetos sin diabetes hubo correlación de los grosores de la capa de fibras nerviosas temporal y de células ganglionares superior ( $r: 0.343 ; p=0.014$ ), nasal inferior ( $r: 0.286 ; p$ $=0.042$ ) y nasal superior ( $r: 0.358 ; p=0.010$ ). En sujetos con diabetes no hubo correlaciones. Conclusión: En sujetos sanos existe correlación entre los grosores de las capas de células ganglionares superior, nasal inferior y nasal superior y el de la capa de fibras nerviosas temporal. En sujetos diabéticos esta correlación se pierde antes de que aparezca retinopatía.
\end{abstract}

Palabras clave: Células ganglionares. Diabetes. Fibras nerviosas. Retina. Tomografía de coherencia óptica.

\section{Abstract}

Objective: To determine the correlation between ganglion cell layer and retinal nerve fiber layer thickness in the temporal sector, in patients with and without diabetes. Design: Observational, retrospective, comparative, and cross-sectional. Material and methods: We studied non-diabetic subjects and diabetic patients without retinopathy, aged 20 to 80 years old. We measured with optical coherence tomography the ganglion cell layer thickness and the temporal nerve fiber layer thickness. We compared the thickness of this layers between groups (Student's t test) and we determined the correlation between variables (Pearson's r). Results: 127 eyes were evaluated, 53 eyes of non-diabetic subjects and 74 of diabetics without retinopathy. The comparison of ganglion cell layer thickness and nerve fiber layer thickness showed no differences between groups. In non-diabetic patients there was a positive correlation between the temporal nerve fiber layer thickness and the

Correspondencia:

*Virgilio Lima-Gómez

E-mail: forscher7@gmail.com
Fecha de recepción: 23-05-2020

Fecha de aceptación: 03-07-2020

DOI: 10.24875/RHJM.20000062
Disponible en internet: 13-11-2020 Rev Hosp Jua Mex. 2020;87(3):117-121

www.revistahospitaljuarez.com 1405-9622/( 2020 Sociedad Médico-Quirúrgica del Hospital Juárez de México, A.C. Publicado por Permanyer. Este es un artículo open access bajo la licencia CC BY-NC-ND (http://creativecommons.org/licenses/by-nc-nd/4.0/). 
ganglion cell layer in the superior ( $r: 0.343 ; p=0.014)$, inferior nasal $(r: 0.286 ; p=0.042)$ and superior nasal sectors ( $r: 0.358$; $p=0.010)$. There were no correlations in diabetic patients. Conclusion: In healthy patients there is a correlation between the temporal nerve fiber layer thickness and the superior, inferior nasal and superior nasal ganglion cell layer thicknesses; these correlations disappear in diabetic patients before diabetic retinopathy develops.

Key words: Diabetes. Ganglion cells. Nerve fiber layer. Optical coherence tomography. Retina.

\section{Introducción}

La retinopatía diabética es la principal causa de ceguera prevenible en pacientes en edad productiva ${ }^{1}$. Es predominantemente una enfermedad vascular, pero la función neural retiniana se altera justo después del comienzo de la diabetes y puede anteceder a lesiones vasculares evidentes ${ }^{2}$. Una las primeras investigaciones sobre neurodegeneración en retinopatía diabética fue de Wolter (en 1961) ${ }^{3}$, quien detectó atrofia de las células ganglionares y degeneración de la membrana limitante interna en ojos donados de pacientes diabéticos. La diabetes mellitus se acompaña de una neuropatía retiniana diabética, esto es, degeneración interna neurorretinal, que se observa estructuralmente como una apoptosis neural, pérdida de células ganglionares, gliosis reactiva y adelgazamiento de la retina interna ${ }^{4}$.

La tomografía de coherencia óptica (TCO) permite obtener imágenes de la retina objetivas, reproducibles y fidedignas para evaluar cuantitativamente el grosor retiniano. La TCO de dominio espectral es una nueva generación de TCO de alta definición que permite analizar imágenes de la neurorretina (capa de células ganglionares, fibras nerviosas y membrana limitante interna) en vivo y podría detectar cambios estructurales en la diabetes antes de la aparición de la retinopatía diabética ${ }^{5}$.

En los pacientes diabéticos tipo 1 se ha encontrado un adelgazamiento generalizado de la capa de fibras nerviosas peripapilares y una disminución del grosor de capas ganglionares en el área macular ${ }^{6}$. En un estudio comparativo del grosor de fibras nerviosas peripapilares (sectorial y general) en pacientes diabéticos sin retinopatía, pacientes prediabéticos y pacientes sin diabetes seleccionados del estudio Maastricht, se encontró en los pacientes con prediabetes un adelgazamiento del sector temporal peripapilar, al compararlos con sujetos sanos ${ }^{7}$.

Otro estudio similar que comparó el grosor de la capa de células ganglionares y de fibras nerviosas en la mácula en sujetos sin diabetes y pacientes con diabetes tipo 2 sin retinopatía, con retinopatía no proliferativa y con retinopatía proliferativa, encontró cambios significativos entre los pacientes con diabetes ( $\sin y$ con retinopatía) y los sujetos sin diabetes en el grosor de la capa de células ganglionares, particularmente en el sector nasal correspondiente al haz maculopapilar. Se planteó que estos hallazgos contribuían a la configuración densa de células ganglionares en la región nasal, en su camino hacia el nervio óptico ${ }^{8}$.

Aunque se miden con el mismo equipo, determinar el grosor de la capa de fibras nerviosas requiere un rastreo distinto a cuantificar el grosor de la capa de células ganglionares; este último solo puede obtenerse en los equipos más recientes. No hay en la literatura actual estudios que demuestren una correlación positiva entre el grosor de la capa de células ganglionares y el del sector temporal de la capa de fibras nerviosas en pacientes con diabetes sin retinopatía. Se realizó un estudio para buscar esa correlación que, en caso de existir, permitiría inferir la disminución del grosor de células ganglionares únicamente con solicitar un estudio de grosor de la capa de fibras nerviosas.

\section{Material y métodos}

Estudio observacional, comparativo, transversal y retrospectivo en sujetos sanos y diabéticos de la Ciudad de México y su área metropolitana. La muestra se obtuvo de los pacientes atendidos en un hospital federal de referencia, entre el 27 de enero y el 31 de enero del 2020. El estudio fue autorizado por las comisiones de investigación y ética en investigación de la institución donde se desarrolló, con número de registro HJM 0752/20-R.

Se incluyeron sujetos de 20 a 80 años, de cualquier sexo, sanos y diabéticos sin retinopatía, con transparencia de los medios oculares que permitiera obtener un estudio de TCO del grosor de células ganglionares y de la capa de fibras nerviosas de adecuada calidad, y que aceptaron participar en el estudio mediante consentimiento informado por escrito. Se eliminaron los sujetos en quienes la calidad del estudio no fue adecuada.

A todos los sujetos se les midió el grosor de la capa de células ganglionares en los sectores superior, temporal superior, temporal inferior, inferior, nasal inferior y nasal superior, así como el grosor de la capa de fibras nerviosas. En todos se obtuvieron dichas 
Tabla 1. Comparación grosor de células ganglionares y capa de fibras nerviosas temporal entre grupos

\begin{tabular}{|l|c|c|c|}
\hline Variable & $\begin{array}{c}\text { Grupo 1 n= 53 } \\
\text { (promedio } \pm \text { DE) }\end{array}$ & $\begin{array}{c}\text { Grupo 2 n= 74 } \\
\text { (promedio } \pm \text { DE) }\end{array}$ & p \\
\hline $\begin{array}{l}\text { Células ganglionares } \\
\text { superior }\end{array}$ & $79.9 \pm 8.0 \mu \mathrm{m}$ & $80.1 \pm 10.4 \mu \mathrm{m}$ & 0.903 \\
\hline $\begin{array}{l}\text { Células ganglionares } \\
\text { temporal superior }\end{array}$ & $80.2 \pm 6.1 \mu \mathrm{m}$ & $78.4 \pm 9.3 \mu \mathrm{m}$ & 0.195 \\
\hline $\begin{array}{l}\text { Células ganglionares } \\
\text { temporal inferior }\end{array}$ & $79.9 \pm 6.3 \mu \mathrm{m}$ & $79.7 \pm 8.4 \mu \mathrm{m}$ & 0.877 \\
\hline $\begin{array}{l}\text { Células ganglionares } \\
\text { inferior }\end{array}$ & $76.0 \pm 8.8 \mu \mathrm{m}$ & $76.7 \pm 9.3 \mu \mathrm{m}$ & 0.684 \\
\hline $\begin{array}{l}\text { Células ganglionares } \\
\text { nasal inferior }\end{array}$ & $79.3 \pm 8.4 \mu \mathrm{m}$ & $79.2 \pm 9.0 \mu \mathrm{m}$ & 0.941 \\
\hline $\begin{array}{l}\text { Células ganglionares } \\
\text { nasal superior }\end{array}$ & $82.5 \pm 8.2 \mu \mathrm{m}$ & $81.4 \pm 9.6 \mu \mathrm{m}$ & 0.506 \\
\hline $\begin{array}{l}\text { Capa de fibras } \\
\text { nerviosas, sector } \\
\text { temporal }\end{array}$ & $79.1 \pm 26.6 \mu \mathrm{m}$ & $81.8 \pm 22.8 \mu \mathrm{m}$ & 0.555 \\
\hline
\end{tabular}

DE: desviación estándar.

mediciones por un solo observador, con el equipo de TCO Cirrus ${ }^{\circledR}$ (Zeiss). El grosor de las capas se midió automáticamente de acuerdo con los algoritmos del equipo, los pacientes sin diabetes se asignaron al grupo 1 y los pacientes con diabetes al grupo 2 .

Las variables en el estudio fueron el grosor de capas de células ganglionares y el de la capa de fibras nerviosas en el sector temporal. Como variables basales se registraron la edad, el volumen macular y grosor del campo central. Se compararon los promedios del grosor de la capa de células ganglionares y de la capa de fibras nerviosas entre grupos mediante la prueba $t$ de Student para medias independientes. Se consideró como diferencia significativa un valor de $p<0.05$; posteriormente se determinó la correlación entre los valores de ambas capas, para lo cual se usó el coeficiente $r$ de Pearson. La información se almacenó y analizó con el programa SPSS ${ }^{\circledR}$ para Windows ${ }^{\circledR}$.

\section{Resultados}

Se evaluaron 127 ojos, 53 ojos del grupo de pacientes sanos y 74 de pacientes con diabetes sin retinopatía. La edad promedio del grupo 1 fue 45.4 años \pm desviación estándar (DE) 16.3, en el grupo 2 la edad promedio fue $55.7 \pm 10.6(p<0.001)$. El promedio de
Tabla 2. Correlaciones entre grosor de capa de células ganglionares y capa de fibras nerviosas temporal en sujetos sin diabetes

\begin{tabular}{|l|c|c|}
\hline Variable & r & p \\
\hline Células ganglionares superior & 0.343 & 0.014 \\
\hline Células ganglionares temporal superior & 0.099 & 0.491 \\
\hline Células ganglionares temporal inferior & 0.200 & 0.160 \\
\hline Células ganglionares inferior & 0.256 & 0.070 \\
\hline Células ganglionares nasal inferior & 0.286 & 0.042 \\
\hline Células ganglionares nasal superior & 0.358 & 0.010 \\
\hline
\end{tabular}

Tabla 3. Correlaciones en pacientes diabéticos

\begin{tabular}{|l|c|c|}
\hline Variable & $\mathbf{r}$ & $\mathbf{p}$ \\
\hline Células ganglionares superior & 0.145 & 0.221 \\
\hline Células ganglionares temporal superior & 0.160 & 0.175 \\
\hline Células ganglionares temporal inferior & 0.166 & 0.162 \\
\hline Células ganglionares inferior & 0.155 & 0.189 \\
\hline Células ganglionares nasal inferior & 0.109 & 0.360 \\
\hline Células ganglionares nasal superior & 0.091 & 0.442 \\
\hline
\end{tabular}

volumen macular en el grupo 1 fue de $9.9 \pm 0.49 \mathrm{~mm}^{3}$ $y$ en el grupo 2 de $12.2 \pm 13.8 \mathrm{~mm}^{3}(p=0.58)$; el grosor de campo central en el grupo 1 fue de $250.4 \pm 21.3 \mu \mathrm{m}$ y en el grupo de $249.0 \pm 26.5 \mu \mathrm{m}$, sin diferencia significativa $(p=0.158)$.

La comparación del grosor de células ganglionares y la capa de fibras nerviosas temporal entre grupos se muestra en la tabla 1. No se encontró diferencia significativa en ninguna de las mediciones.

Las correlaciones del grosor de la capa de células ganglionares y la capa de fibras nerviosas temporal en sujetos sin diabetes se muestran en la tabla 2 . Se encontró una correlación positiva entre el grosor de los sectores de células ganglionares superior ( $r$ : 0.343; $p=0.014$ ), el sector nasal inferior ( $r: 0.286 ; p=0.042$ ) y el sector nasal superior ( $r: 0.358 ; p=0.010$ ) y el grosor de la capa de fibras nerviosas temporal.

Las correlaciones entre el grosor de la capa de células ganglionares y el grosor de la capa de fibras nerviosas temporal en sujetos con diabetes se muestran en la tabla 3. No se encontró correlación entre el grosor de alguno de los sectores de células ganglionares y el grosor de la capa de fibras nerviosas temporal. 


\section{Discusión}

Se encontró en sujetos sin diabetes una correlación entre el grosor de la capa de fibras nerviosas en el sector temporal y el grosor de la capa de células ganglionares en tres sectores: superior ( $r: 0.343 ; p=0.014$ ), nasal inferior ( $r: 0.286 ; p=0.042)$ y nasal superior ( $r: 0.358 ; p=0.010$ ). Las correlaciones encontradas en sujetos sin diabetes no se presentaron en pacientes diabéticos.

Los axones de las células ganglionares conforman la capa de fibras nerviosas. El sector temporal de la capa de fibras nerviosas peripapilares se distingue del resto de los sectores porque recibe al haz papilomacular, que únicamente contiene los axones de las células ganglionares de la mácula. El grosor de la capa de fibras nerviosas peripapilares ha podido medirse desde versiones anteriores de TCO, pero el grosor de la capa de células ganglionares está disponible solo con los modelos de dominio espectral; por ello se han estudiado ampliamente los cambios en el grosor de fibras nerviosas peripapilares, pero solo recientemente se ha buscado una correlación entre esa variable y la disminución del grosor de la capa de células ganglionares.

En pacientes con glaucoma y glaucoma preperimétrico se ha identificado una correlación entre la disminución del grosor de la capa de células ganglionares y el de las fibras nerviosas sin enfatizar algún sector en específico ${ }^{9}$; en el glaucoma se pierden fibras nerviosas en toda la región peripapilar, ya que es una enfermedad que lleva a la atrofia óptica.

Existen estudios que han comparado los grosores de la capa de fibras nerviosas y el de la capa de células ganglionares en pacientes sin diabetes y diabéticos sin retinopatía que encontraron una disminución en el segundo grupo de ambos grosores, pero no se enfocaron en el sector temporal de las fibras nerviosas ${ }^{2,10}$. Otro estudio encontró una disminución del grosor de la capa de células ganglionares en el área pericentral macular con una disminución secundaria del grosor de fibras nerviosas en el área periférica macular, que se atribuyó a la disposición de las fibras en el área nasal?. Ninguno de estos reportes describió la correlación entre el grosor de la capa de células ganglionares en la mácula y la región temporal peripapilar que recibe sus axones.

A diferencia del glaucoma, la retinopatía diabética no evoluciona hacia la atrofia óptica; sin embargo, en ojos con esta enfermedad se ha reportado una disminución del grosor de la capa de células ganglionares en el área macular, que se ha atribuido a daño neural, asociado o no con los cambios vasculares. Por ello se consideró que un cambio temprano del grosor de la capa de células ganglionares maculares no afectaría a todas las fibras nerviosas peripapilares, sino solo al sector temporal.

En nuestro estudio no se encontró una diferencia del grosor de la capa de células ganglionares entre los grupos, lo que indica que la diabetes aún no había causado una afección neural que pudiera detectarse por TCO, como la que se presenta cuando ya existe retinopatía. Llama la atención que las correlaciones encontradas en sujetos sin diabetes no se presentaron en pacientes diabéticos, este cambio puede representar una alteración temprana, pero como se identificó en ojos sin cambios estructurales requerirá evaluarse en conjunto con pruebas funcionales.

Se ha propuesto que la diabetes puede causar una degeneración temprana en la retina neurosensorial; si este cambio iniciara por una disminución del grosor de la capa de células ganglionares, se requeriría medirlas directamente, ya que no existe correlación entre esa variable y el grosor temporal de la capa de fibras nerviosas.

\section{Conclusión}

En pacientes diabéticos no se encontró correlación entre el grosor de la capa de fibras nerviosas en el sector temporal y el grosor de la capa de células ganglionares. Los cambios de esta última estructura requieren medirse directamente.

\section{Financiamiento}

La presente investigación no ha recibido ayudas específicas provenientes de agencias del sector público, sector comercial o entidades sin ánimo de lucro.

\section{Conflicto de intereses}

Los autores declaran no tener conflicto de intereses alguno.

\section{Responsabilidades éticas}

Protección de personas y animales. Los autores declaran que para esta investigación no se han realizado experimentos en seres humanos ni en animales. 
Confidencialidad de los datos. Los autores declaran que en este artículo no aparecen datos de pacientes.

Derecho a la privacidad y consentimiento informado. Los autores han obtenido el consentimiento informado de los pacientes y/o sujetos referidos en el artículo. Este documento obra en poder del autor de correspondencia.

\section{Bibliografía}

1. Wong TY, Sun J, Kawasaki R, Ruamviboonsuk P, Gupta N, Lansingh VC et al. Guidelines on diabetic eye care: The International Council of Ophthalmology Recommendations for screening, follow-up, referral, and treatment based on resource settings. Ophthalmology. 2018;125:1608-22.

2. Carpineto $P$, Toto $L$, Aloia R, Ciciarelli V, Borrelli E, Vitacolonna E, et al. Neuroretinal alterations in the early stages of diabetic retinopathy in patients with type 2 diabetes mellitus. Eye (Lond). 2016;30:673-9.
3. Barber AJ. A new view of diabetic retinopathy: a neurodegenerative disease of the eye. Prog Neuropsychopharmacol Biol Psychiatry. 2003;27:283-90.

4. Sohn EH, van Dijk HW, Jiao C, Kok PH, Jeong W, Demirkaya N, et al Retinal neurodegeneration may precede microvascular changes characteristic of diabetic retinopathy in diabetes mellitus. Proc Natl Acad Sci U S A. 2016:113:2655-64.

5. Lynch SK, Abràmoff MD. Diabetic retinopathy is a neurodegenerative disorder. Vision Res. 2017;139:101-7.

6. Gundogan FC, Akay F, Uzun S, Yolcu U, Ca冈lltay E, Toyran S. Early neurodegeneration of the inner retinal layers in type 1 diabetes mellitus. Ophthalmologica. 2016;235:125-32.

7. Chhablani J, Sharma A, Goud A, Peguda HK, Rao HL, Begum VU, et al. Neurodegeneration in type 2 diabetes: Evidence from spectral-domain optical coherence tomography. Invest Opthalmol Vis Sci. 2015;56:6333-8.

8. De Clerck EEB, Schouten JSAG, Berendschot TTJM, Beckers HJM, Schaper NC, Schram MT, et al. Loss of temporal peripapillary retinal nerve fibers in prediabetes or type 2 diabetes without diabetic retinopathy: The Maastricht Study. Invest Ophthalmol Vis Sci. 2017;58:1017-27.

9. Oli A, Joshi D. Can ganglion cell complex assessment on cirrus HD OCT aid in detection of early glaucoma? Saudi J Ophthalmol. 2015;29:201-4.

10. Ng DS, Chiang PP, Tan G, Cheung CG, Cheng CY, Cheung CY, et al. Retinal ganglion cell neuronal damage in diabetes and diabetic retinopathy. Clin Exp Ophthalmol. 2016;44:243-50. 